\title{
Retracted Article: Residues and dissipation dynamics of spirodiclofen in apple and soil
}

\author{
Xiaosha Gao · Yun Bai · Hongyan Zhang • \\ Pengjun Xu • Lifen Wu
}

Received: 29 December 2008 / Accepted: 4 June 2009 / Published online: 20 June 2009

(C) Springer Science + Business Media B.V. 2009

After publication, the authors discovered some errors in the original data, making the results and conclusions incorrect. They have requested retraction of the article. For more information, please contact the original corresponding author: Hongyan Zhang, Email: hongyan@cau.edu.cn

After publication, the authors discovered some errors in the original data, making the results and conclusions incorrect. They have requested retraction of the article. For more information, please contact the original corresponding author: Hongyan Zhang, Email: hongyan@cau.edu.cn.

X. Gao · Y. Bai · H. Zhang $(\varangle)$

College of Science, China Agricultural University,

Beijing, 100193, People's Republic of China

e-mail: hongyan@cau.edu.cn

P. Xu

National Research Center for Environmental

Analysis and Measurements,

Beijing, 100029, People's Republic of China

L. Wu

Institute for the Control of Agrochemicals

of Hebei Province, Shijiazhuang, 050031,

Hebei Province, People's Republic of China 